\title{
Proteomic analysis of NMDA receptor-adhesion protein signaling complexes
}

\author{
H olger Husi ${ }^{1}$, M alcolm A. Ward ${ }^{2}$, Jyoti S. Choudhary², Walter P. Blackstock² and Seth G. N. Grant ${ }^{1}$ \\ ${ }^{1}$ Centre for Genome Research, Centre for Neuroscience, University of Edinburgh, West M ains Road, Edinburgh EH 9 3JQ, UK \\ 2 Glaxo Wellcome Research and D evelopment Ltd, Gunnels Wood Road, Stevenage SG 1 2NY, UK \\ Correspondence should beaddressed to S.G.N.G. (seth.grant@ed.ac.uk)
}

\begin{abstract}
$\mathrm{N}$-methyl-D-aspartate receptors (NMDAR) mediate long-lasting changes in synapse strength via downstream signaling pathways. We report proteomic characterization with mass spectrometry and immunoblotting of NMDAR multiprotein complexes (NRC) isolated from mouse brain. The NRC comprised 77 proteins organized into receptor, adaptor, signaling, cytoskeletal and novel proteins, of which 30 are implicated from binding studies and another 19 participate in NMDAR signaling. NMDAR and metabotropic glutamate receptor subtypes were linked to cadherins and L1 cell-adhesion molecules in complexes lacking AM PA receptors. These neurotransmitteradhesion receptor complexes were bound to kinases, phosphatases, GTPase-activating proteins and Ras with effectors including M APK pathway components. Several proteins were encoded by activity-dependent genes. Genetic or pharmacological interference with 15 NRC proteins impairs learning and with 22 proteins alters synaptic plasticity in rodents. Mutations in three human genes (NF1, Rsk-2, L1) are associated with learning impairments, indicating the NRC also participates in human cognition.
\end{abstract}

A prevailing cellular model of learning involves modification of synapse strength, induced by patterns of neuronal activity during training, which encodes information in neural networks ${ }^{1,2}$. Understanding the molecular mechanisms used by synapses to detect altered patterns of firing, and how signaling cascades then change the strength of synaptic transmission, may provide basic insights into this model and illuminate pathological mechanisms that occur in human learning impairments and other psychiatric disorders 3 . Considerable attention has focused on the N M DA subtype of glutamate receptor ${ }^{4}$ because its blockade in the hippocampus impairs both synaptic plasticity and learning. This receptor channel, which allows calcium influx into the postsynaptic spine, regulates kinases, phosphatases and other enzymes, which then regulate AM PA ( $\alpha$-amino-3-hydroxy-5-methyl-4isoxasole-4-propionic acid) glutamate receptors (AM PARs), spine cytoskeletal changes, translation, transcription and other events. These diverse actions indicate the N M DAR couples to multiple intracellular signaling pathways. M oreover, in a physiological setting, these signals must be subtly integrated, as shown by electrophysiological studies in CA3-CA1 synapses of the hippocampus, where low-frequency synaptic stimulation results in N M DAR-dependent long-term depression (LTD) and higher frequencies in long-term potentiation (LTP) of synaptic strength?2.

LTP comprises a series of temporally distinct processes that can broadly be separated into 'induction' and 'maintenance' phases. The induction phase includes the train of stimuli that activate the N M DA receptor, which lasts for seconds or less, and a period of minutes during which second messenger pathways act, leading to a new stable level of synapse strength in the maintenance phase. Unlike the induction phase, the maintenance phase is resistant to inhibitors of second messengers, such as kinases and phosphatases, although it is sensitive to inhibitors of RNA and protein synthesis for the first two to three hours, indicating a role for new gene expression ${ }^{1,2}$. In parallel with this requirement for gene expression in the late phases of LTP, the NM DAR is required for transcriptional activation and dendritic trafficking of mRNAs and proteins such as Arg3.1/Arc and Homer/Vesl. A recent review ${ }^{5}$ of the molecular mechanisms of LTP highlighted the large number of implicated molecules and the lack of a satisfying model. In addition to neurotransmitter receptors and multiple signaling proteins, this list of molecules included adhesion and structural proteins. An approach to the problem of understanding the molecular basis of LTP and its signaling pathways is to combine a functional genetic and pharmacological dissection with structural analysis of the organization of synaptic molecules.

Assembly of receptors with signal transduction proteins into large multiprotein complexes has emerged as a general mechanism of cellular signaling ${ }^{6}$. The NM DAR binds postsynaptic proteins ${ }^{7-9}$, including postsynaptic density 95 (PSD -95), which regulates synaptic plasticity in mutant mice ${ }^{10}$, consistent with the hypothesis that a signaling complex regulates synaptic plasticity and learning. To further explore this model, we have isolated NM DAR-PSD-95 complexes from mouse brain and analyzed their properties using proteomic techniques, which are powerful tools for analysis of large protein complexes ${ }^{11,12}$. This approach allows the detection of protein interactions that require posttranslational modifications such as phosphorylation (for example, SH 2 binding of PI3-kinase to NM DAR subunits ${ }^{13}$ ) and lipid modification (for instance, in PSD-95; ref. 14) and ternary or 


\section{articles}

a

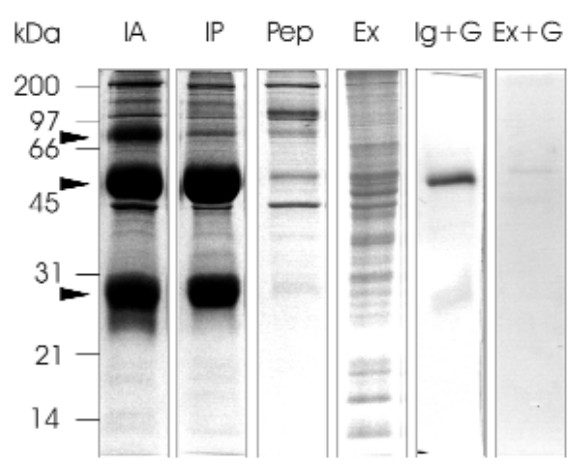

b

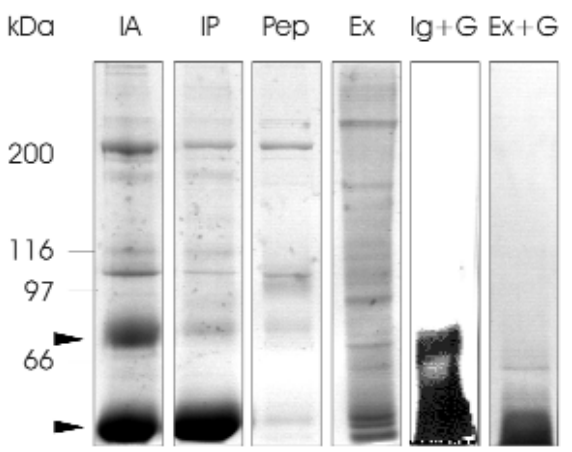

C

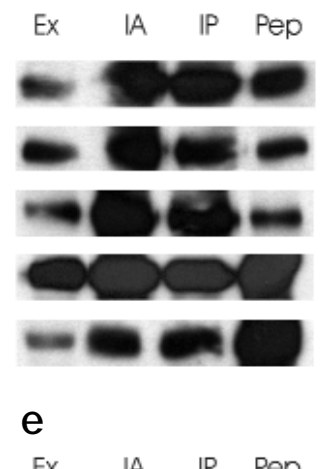

NR1

NR2A

NR2B

PSD95

Chapsyn 10
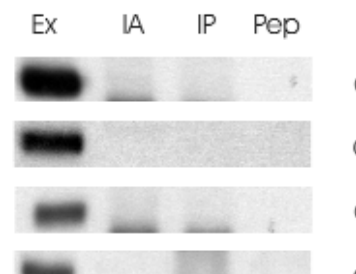

GluR1

GluR2

GluR2/3

GluR4

GRIP

f
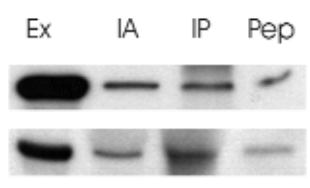

d

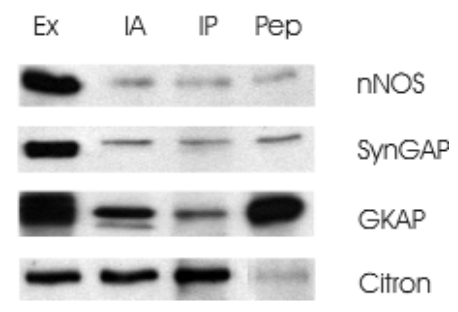

g

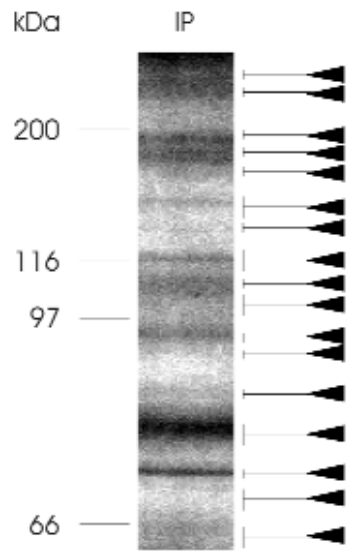

Fig. 1. Basic composition of $N R C$ isolated from mouse brain. (a, b) Protein staining of SD S-PAGE (a, 12\%; b, 6\%) from immunoaffinity (IA), immunoprecipitation (IP) and N R2B peptide affinity (Pep) isolation of N MDAR from mouse brain extracts (Ex). N egative controls are MAP-N R1 Ig bound to protein $\mathrm{G}$-sepharose $(\mathrm{Ig}+\mathrm{G})$ and extracts bound to protein $\mathrm{G}$-sepharose $(E x+G)$. Arrowheads indicate position of Ig species. A complex mixture of specific binding proteins were observed, mainly in the range above $70 \mathrm{kD}$, and were analyzed by mass spectrometry (g: Table 2). (c) Immunoblot analysis of N MDAR subunits and MAGUK proteins in NRC. NR1, NR2A, NR2B, PSD -95 and Chapsyn-110 were detected. The N RC from 5 mg of starting material, $50 \mu \mathrm{g}$ of protein loaded in total extract (Ex), was isolated and loaded per lane (IA, IP, Pep). Y ield and enrichment cannot be calculated because the IA, IP and Pep samples contained interfering Ig and peptide, and thus did not permit protein quantitation. (d) Immunoblot analysis of PSD -95 binding proteins in N RC. W e detected nN O S, SynG AP, GKAP and Citron. (e) Immunoblot analysis of AMPA receptor subunits and GRIP in NRC. GluR1, GluR2, GluR2/3, GluR4 and GRIP were readily detected in the starting material (Ex) but not in the three NRC preparations. (f) Immunoblot analysis of $\mathrm{mGluR} 1$ receptor and Homer proteins in N RC. We detected mGluRl $\alpha$ and Homer, indicating that the NRC includes metabotropic glutamate receptors but not AMPA receptors. (g) Protein staining of N RC (6\% SD S-PAGE) showing position (arrowheads) of bands excised for mass spectrometry.

weak interactions undetected by 2-hybrid screen ${ }^{11}$, which have been extensively used to identify NM DAR and PSD-95 binding partners ${ }^{7-9}$. M oreover, biochemical characterization of NRC from brain is required for analysis of mice carrying mutations affecting the complexes as well as other in vivo analyses.

Developments in high-sensitivity mass spectrometry, particularly nano-el ectrospray mass spectrometry coupled with search engines to access the huge amount of data produced by the genome sequencing projects, have fueled the growth of proteomics ${ }^{15}$. Initially defined as the protein complement of a genome, the term proteome has been generalized to encompass any large-scale approach to protein characterization. Proteins may exist as discrete known proteins in databases (such as SwissProt), as complete or partial sequences in EST databases (for example, dbEST), or as putative open reading frames in genome databases. All three are accessible to searching by combinations of mass and partial sequence information generated by mass spectrometry, searching the protein databases directly and translating the DNA sequences in six frames. The required numerical search attributes are generated by specific proteolytic digestion of the protein, and subsequent analysis of the resulting peptide pool by mass spectrometry. Two measurements are usually made; the first is the mass of as many peptides as possible. This peptide mass fingerprint (PM F) alone may be sufficient to identify the original protein, particularly if the protein was isolated in a relatively pure state by, for example, two-dimensional gel electrophoresis. More often though, proteins are available only as mixtures, and here PM Fs are less use ful. In this case, mass and partial sequence information are generated by tandem mass spectrometry (MS/MS) and combined to provide powerful discriminating input for mass spectrometry search engines. This latter approach is sufficiently robust to deal with simple mixtures, and if combined with on-line separation techniques can deal with very complex protein mixtures, for example, from bands from one-dimensional gels, or even total protein complexes. This is a more appropriate approach for searching EST databases, which rarely contain full-length protein sequences. 
a

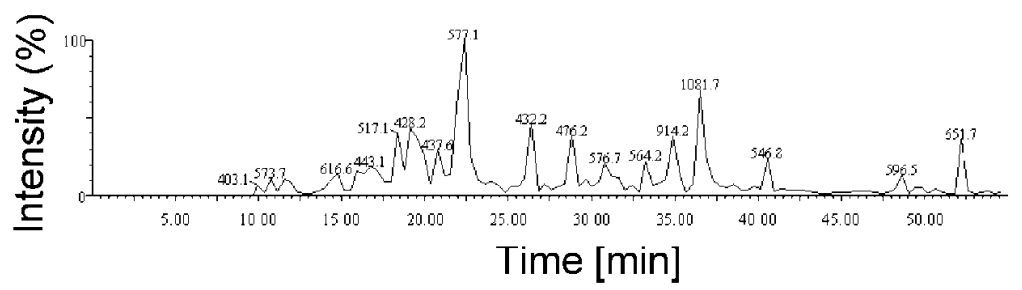

b

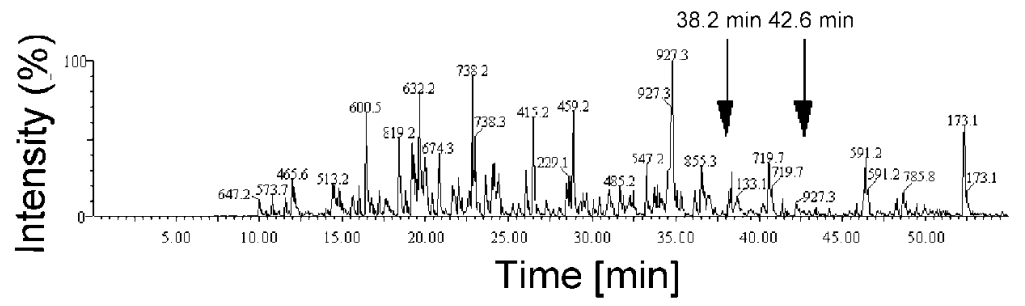

C

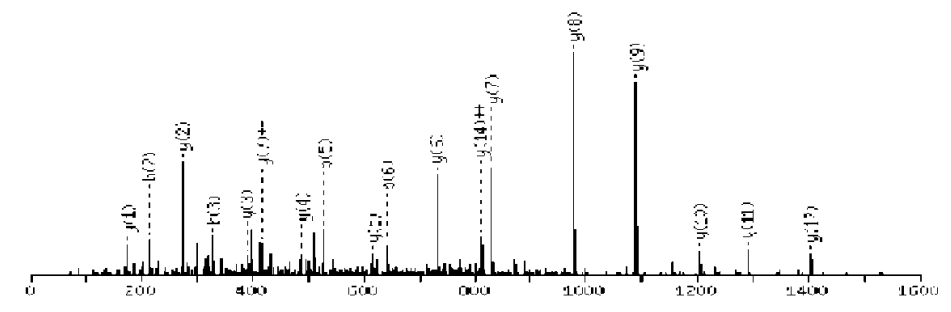

d

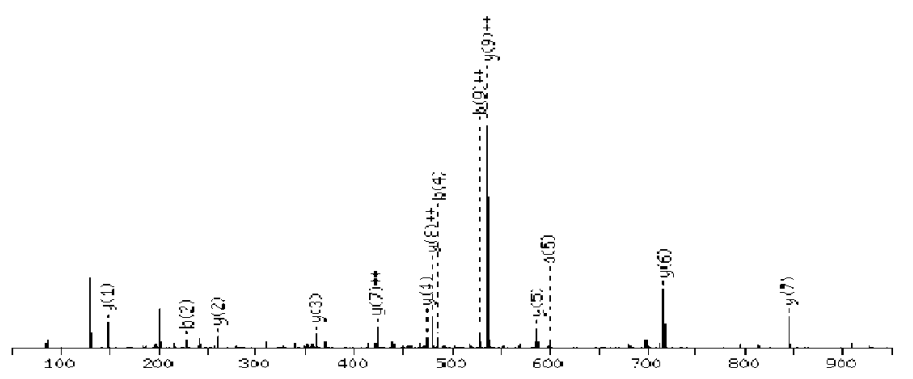

Fig. 2. Mass spectrometry analysis of the NRC. (a) Base peak chromatograms generated in MS survey mode (example from trypsin digestion products of a $90 \mathrm{kD}$ protein band) The two most intense ions in each MS scan are automatically selected for collision-induced fragmentation and analysis in MS/MS mode (LC-MS/MS; b). (b) Base peak chromatograms of one of the two MS/MS channels. The labels at $38.2 \mathrm{~min}$ and $42.6 \mathrm{~min}$ correlate with two peptides that were selected to generate peptide sequence information. (c, d) Corresponding MS/MS spectra obtained from the precursors eluting at these times. (c) Averaged MS/MS spectrum obtained from peak eluting at $42.6 \mathrm{~min}$ in (b). A doubly charged ion at mass to charge ratio $(\mathrm{m} / \mathrm{z}) 809.72$, corresponding to a peptide of $1617.44 \mathrm{D}$, was selected for fragmentation by collision-induced dissociation. The fragment ions that originate either from the $\mathrm{N}$ terminus (b type ions) or the $\mathrm{C}$ terminus ( $y$ " type ions) correspond to an aminoacid sequence that was identified using the Mascot database search program. In this instance, the MS/MS spectrum matched the peptide sequence VN DSILFVN EVDVR from PSD - 95 residues 113-126 (accession number Q 62108). (d) Example of a novel NRC protein identified from the averaged MS/MS spectrum obtained from the peak eluting at $38.2 \mathrm{~min}$ in (b). The observed doubly charged precursor ion at $\mathrm{m} / \mathrm{z}$ 538.68 , relating to a $1071.65 \mathrm{D}$ peptide, was identified by Mascot search program as the sequence DLKEILTLK, which matches APPL, an adaptor protein for PI3 kinase and AKT/PKB kinase ${ }^{29}$.

blocked affigel-10 used in antibody and peptide affinity purification (Fig. 1), as well as other peptides and antibodies (data not shown). The integrity of the complexes and specificity of protein interactions were examined. NMDAR subunits (NR1, NR2A, $N R 2 B$ ) and their reported interacting proteins7-9 (PSD-95, Chapsyn-110/PSD-93, calmodulin, $\alpha$-actinin 2, calcium/calmodulin kinase II (CaM KII), phospholipase C $\gamma$ ) were readily detected (Fig. 1c and Table $\mathbf{1}$ ), and PSD - 95 binding proteins including nN OS, SynGAP, SAPAP/GKAP and Citron were also found (Fig. 1d). We next examined AM PA subunits (GluR1-4) and their cognate adapter protein GRIP16, which were undetected in the NRC despite their abundance in the extract (Fig. le). Similarly, we did not detect N M DAR complex proteins in immunoprecipitations with AM PAR-specific antibodies (data not shown). Kainate receptor subunits (GluR6/7) were detected, consistent with reports that PSD-95 binds these subunits ${ }^{17}$. The metabotropic ( $m G l u R 1 \alpha$ ) receptors and their cognate binding partner Homer/Vesl, were found in the NRC (Fig. 1f), consistent with reports from 2-hybrid screening that $\mathrm{H}$ omer binds Shank, which can bind GKAP to PSD-95 (refs. 18, 19). Therefore the NM DAR and mGluR receptors are associated in distinct complexes from AM PA receptors.

We next identified novel components of the NRC using two strategies: a western blotting screen for candidate proteins involved 
Table 1. Summary of molecular composition of the NRC.

\begin{tabular}{|c|c|c|c|c|c|c|c|c|}
\hline & Protein & $\operatorname{Mr}(\mathbf{k D})$ & Ex & IA & IP & Pep & $\mathbf{A b}$ & Binding partner \\
\hline \multicolumn{9}{|c|}{ Glutamate receptors } \\
\hline 1 & NR1 & 120 & +++ & +++ & +++ & +++ & a & $2,3,18,35,68,95,96,101$ \\
\hline 2 & NR2A & 180 & +++ & +++ & +++ & +++ & $b$ & $1,3,10,11,12,35,73,96$ \\
\hline 3 & NR2B & 180 & +++ & +++ & +++ & +++ & c & $1,2,10,11,12,35,43,95,71,73,96,101$ \\
\hline 4 & GluR1 & 108 & +++ & - & - & - & $d$ & \\
\hline 5 & GluR2 & 102 & +++ & - & - & - & $d$ & \\
\hline 6 & GluR2/3 & 102 & +++ & - & - & - & e & \\
\hline 7 & GluR4 & 108 & +++ & - & - & - & $d$ & \\
\hline 8 & GluR6 + 7 & 117 & +++ & + & + & + & $b$ & 10,12 \\
\hline 9 & $\operatorname{mGluR} 1 \alpha$ & 200 & +++ & +++ & +++ & ++ & $d$ & $15,68,73,101$ \\
\hline \multicolumn{9}{|c|}{ Scaffolding and adaptors } \\
\hline 10 & PSD -95 & 95 & +++ & +++ & +++ & +++ & $f$ & $2,3,8,10,11,12,13,65,69,75$ \\
\hline 11 & ChapSyn110 / PSD -93 & 110 & +++ & +++ & +++ & +++ & $g$ & $2,3,10,69$ \\
\hline 12 & Sap102 & 115 & +++ & + & + & +++ & $\mathrm{h}$ & $2,3,8,10,13,65,68$ \\
\hline 13 & GKAP / SAPAP & $95-140$ & +++ & +++ & ++ & +++ & e & $10,12,14$ \\
\hline 14 & Shank & 200 & +++ & +++ & +++ & +++ & $\mathrm{i}$ & $13,15,102$ \\
\hline 15 & Homer & $28 / 45$ & +++ & ++ & ++ & ++ & j & $9,15,14$ \\
\hline 16 & GRIP & 120 & +++ & - & - & - & e & \\
\hline 17 & ABP (G RIP2) & $95-130$ & +++ & - & - & - & $\mathrm{k}$ & \\
\hline 18 & Yotiao & 200 & +++ & +++ & +++ & +++ & I & $1,25,37$ \\
\hline 19 & AKAP150 & 150 & +++ & +++ & +++ & +++ & I & $25,26-34,39,68$ \\
\hline 20 & N SF & 83 & +++ & ++ & ++ & +++ & $\mathrm{m}$ & 5,20 \\
\hline$\overline{\text { PKA }}$ & & & & & & & & $18,19,92$ \\
\hline 21 & PKA catalytic subunit & 40 & +++ & ++ & ++ & ++ & $f$ & \\
\hline 22 & PKA-R $1 \alpha$ and $\beta$ & 48 & +++ & $c h$ & $c h$ & - & $f$ & \\
\hline 23 & PKA-R1a- $\alpha$ & 49 & +++ & ch & ch & - & $f$ & \\
\hline 24 & PKA-R2 $\alpha$ & 51 & +++ & $c h$ & ch & - & $f$ & \\
\hline 25 & PKA-R2 $\beta$ & 53 & +++ & ++ & ++ & ++ & $f$ & \\
\hline$\overline{\text { PKC }}$ & & & & & & & & 19,105 \\
\hline 26 & PKC $\alpha$ & 82 & +++ & - & - & - & $f$ & \\
\hline 27 & PKC $\beta$ & 80 & +++ & ++ & ++ & ++ & $f$ & \\
\hline 28 & PKC $\gamma$ & 80 & +++ & +++ & +++ & +++ & $f$ & \\
\hline 29 & PKC $\delta$ & 78 & +++ & - & - & - & $f$ & \\
\hline 30 & $\mathrm{PKC} \varepsilon$ & 90 & +++ & ++ & ++ & ++ & $f$ & \\
\hline 31 & PKC $\eta$ & 82 & +++ & - & - & - & $f$ & \\
\hline 32 & PKC $\theta$ & 79 & +++ & - & - & - & $f$ & \\
\hline 33 & PKC 1 & 74 & +++ & - & - & - & $f$ & \\
\hline 34 & $\mathrm{PKC} \lambda$ & 74 & +++ & - & - & - & $f$ & \\
\hline \multicolumn{3}{|c|}{ CaM kinase } & & & & & & $1,2,3,65,68,69$ \\
\hline 35 & CaM kinase II $\beta$ & 60 & +++ & +++ & +++ & +++ & $f$ & \\
\hline 36 & phospho-C aM kinase II & 60 & +++ & ++ & ++ & ++ & $n$ & \\
\hline
\end{tabular}

\section{Phosphatases}

\begin{tabular}{|c|c|c|c|c|c|c|c|c|}
\hline \\
\hline 37 & PP1 & 36 & +++ & +++ & +++ & +++ & $f$ & \multirow{2}{*}{18} \\
\hline 38 & PP2A & 36 & +++ & +++ & +++ & + & $f$ & \\
\hline 39 & PP2B (calcineurin) & 61 & +++ & + & + & + & $f$ & \multirow[t]{4}{*}{19} \\
\hline 40 & PP5 & 50 & +++ & ++ & ++ & ++ & 0 & \\
\hline 41 & PTP1B & 50 & +++ & ch & ch & - & $b$ & \\
\hline 42 & PTP1C & 68 & +++ & - & - & - & $b$ & \\
\hline 43 & PTP1D/SHP2 & 72 & +++ & ++ & ++ & ++ & $f$ & 3 \\
\hline \multicolumn{9}{|c|}{ Tyrosine kinases } \\
\hline 44 & Src & 60 & +++ & + & + & + & $p$ & \multirow[t]{3}{*}{$47,59,60,90,101$} \\
\hline 45 & Fyn & 59 & +++ & - & - & - & $f$ & \\
\hline 46 & FAK & 125 & +++ & - & - & - & $f$ & \\
\hline 47 & PYK2 & 116 & +++ & + & + & + & $f$ & 44 \\
\hline \multicolumn{9}{|c|}{ MAP kinase pathway } \\
\hline 48 & ERK (pan ERK) & $42 / 44$ & +++ & ++ & ++ & ++ & $f$ & \multirow{5}{*}{57} \\
\hline 49 & ERK 1 & $42 / 44$ & +++ & ++ & ++ & ++ & $f$ & \\
\hline 50 & ERK2 & 42 & +++ & ++ & ++ & ++ & $f$ & \\
\hline 51 & ERK 3 & 62 & +++ & - & - & - & $f$ & \\
\hline 52 & phospho-ERK $1 / 2$ & $42 / 44$ & +++ & - & - & - & $q$ & \\
\hline
\end{tabular}




\begin{tabular}{llccccccc}
53 & MEK1 & 45 & +++ & ++ & ++ & + & $f$ & 59 \\
54 & MEK2 & 46 & +++ & ++ & ++ & + & $f$ & 59 \\
55 & MKP2 & 43 & +++ & +++ & +++ & ++ & $f$ & \\
56 & JNKK1/MKK4 & 44 & +++ & - & - & - & $f$ & \\
57 & Rsk & 90 & +++ & ++ & ++ & ++ & $f$ & 50 \\
58 & Rsk-2 & 90 & +++ & ++ & +++ & + & $\mathrm{r}$ & 50 \\
59 & C-Raf1 & 74 & +++ & ++ & ++ & + & $\mathrm{f}$ & $44,53-54,60$ \\
\hline
\end{tabular}

\section{Small G-proteins and modulators}

\begin{tabular}{lllllllll}
60 & H-Ras & 21 & +++ & $\mathrm{cl}$ & $\mathrm{cl}$ & + & $\mathrm{f}$ & $44,59,65,66,71$ \\
61 & Rac1 & 21 & +++ & ++ & + & + & $\mathrm{f}$ & $75,101,102$ \\
62 & Rap1 & 21 & +++ & - & - & - & $\mathrm{f}$ & \\
63 & Rap2 & 21 & +++ & ++ & ++ & + & $\mathrm{f}$ & \\
64 & RalA & 24 & +++ & $\mathrm{cl}$ & $\mathrm{cl}$ & + & $\mathrm{f}$ & \\
65 & SynGAP & 135 & +++ & ++ & ++ & ++ & $\mathrm{e}$ & $10,12,35,60$ \\
66 & N F1 & 250 & +++ & + & + & + & $\mathrm{r}$ & 60,101 \\
67 & P120GAP & 120 & +++ & - & - & - & $\mathrm{f}$ & \\
\hline
\end{tabular}

\section{Other signaling molecules}

$\begin{array}{llccccc}68 & \text { Calmodulin } & 15 & +++ & +++ & +++ & +++ \\ 69 & \text { nN OS } & 155 & +++ & ++ & ++ & ++ \\ 70 & \text { eN OS } & 140 & +++ & - & - & - \\ 71 & \text { PI3 kinase } & 85 & +++ & + & + & + \\ 72 & \text { Calpain } & 30 & +++ & - & - & - \\ 73 & \text { PLC } ~ & 150 & +++ & ++ & ++ & ++ \\ 74 & \text { CPLA2 } & 110 & +++ & +++ & ++ & ++ \\ 75 & \text { Citron } & 183 & +++ & +++ & +++ & ++ \\ 76 & \text { VAV } & 95 & +++ & - & - & - \\ 77 & \text { Arg3.1 } & 55 & +++ & +++ & +++ & +++ \\ 78 & \text { CREB/CREM } & 46 / 26 & - & - & - & - \\ 79 & \text { N CK } & 47 & +++ & - & - & - \\ 80 & \text { SHC } & 52 / 66 & +++ & - & - & -\end{array}$

\section{Cell-adhesion and cytoskeletal proteins}

82 E-C adherin

83 P-Cadherin

$84 \quad$ Cadherin-5

$85 \quad$ Desmoglein

$86 \alpha-C$ atenin

$87 \quad \beta$-Catenin

$88 \gamma$-Catenin

$89 \quad$ L1

90 pp120cas

91 Paxillin

92 MAP2B

93 Vinculin

94 Actin

$95 \alpha$-actinin 2

$96 \quad$ Spectrin

97 Myosin (brain)

98 Tau

99 Tensin

100 Ezrin

101 Tubulin

102

103

104

105

106

\section{Cortactin}

CortBP-1

Clathrin heavy chain

Dynamin

H sp-70 t+t $\quad++\quad++\quad+$

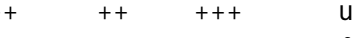

$\begin{array}{lllll}+++ & - & - & - & f \\ +4+ & - & - & - & f\end{array}$

$+++$

$+++$

$+++$

$+++$

$+++$

$+++$

$+++$

+++
+++

$+++$

$+++$

$+++$

$+++$

$+++$

$+++$

$+++$

$+++$

$+++$

$+++$

$+++$

$+++$

$+++$

$+++$

$+++$
$1,9,12,19,35,69,92$

$10,11,35,68$

$3,60,101$

$2,3,9,94,101$

10,61

$81,87,88,90$

85,88

$87,88,95$

81,86

85,86

89

44,81

$25,68,101$

$73,95,96,97,102$

$1,3,86,94$

$1,2,3,94,96$

94

$1,3,9,61,66,71,73,92,96,106$

$14,44,61,94,103$

102

106

26-34

101,104

Immunoblotting screen of NRC and known binding partners within N MDAR complexes. Classes of proteins are boxed, and specific molecular names are indicated with identifying numbers (1-106). Mr, relative molecular mass; Ex, mouse brain extracts; IA, complexes isolated by MAP-N R1 immunoaffinity; IP, complexes isolated by MAP-N R1 immunoprecipitation; Pep, complexes isolated by N R2B peptide affinity; Ab, antibody source (see Methods); binding partner, Identified interactions from published in vitro studies. Specific brain proteins were assayed in three preparations of complexes (IA, IP, Pep) using immunoblotting, and signals were scored as strong $(+++)$, medium $(++)$, weak $(+)$ or undetectable $(-)$. Some proteins could not be analyzed in IA or IP because of comigration of Ig (ch, comigration heavy chain Ig; cl, comigation light chain Ig). The scoring is not suitable for comparison of different proteins because it depends on the properties of specific antibodies, but it is suitable to indicate a relative enrichment from extract. For each protein found in the complexes, the reported associated proteins are indicated (binding partners) by reference to the numbering scheme in the first column. 


\section{articles}

\begin{tabular}{|c|c|c|c|}
\hline Protein name & $\operatorname{Mr}(\mathbf{k D})$ & Accession number & Number of peptides \\
\hline Bassoon & 418 & 088778 & 11 \\
\hline Myosin B heavy chain (non muscle type) & 229 & P35580 & 27 \\
\hline p53 binding protein-1 & 214 & Q 12888 & 2 \\
\hline Tight junction protein ZO -1 & 195 & P39447 & 13 \\
\hline Clathrin heavy chain & 192 & P11442 & 1 \\
\hline NR2B & 166 & Q 01097 & 85 \\
\hline NR2A & 165 & P35436 & 36 \\
\hline SynGAP & 143 & AAC 08071 & 9 \\
\hline NR1 & 105 & P35438 & 208 \\
\hline$\alpha$-actinin 2 & 103 & Q 62744 & 25 \\
\hline Sap97 & 100 & Q 12959 & 2 \\
\hline Hypothetical $97.8 \mathrm{kD}$ protein & 98 & CAB43675 & 3 \\
\hline Dynamin & 96 & Q 61358 & 4 \\
\hline Chapsyn 110 & 95 & Q 63622 & 108 \\
\hline Sap102 & 93 & P70175 & 9 \\
\hline Phosphofructokinase & 86 & P12382 & 7 \\
\hline N SF vesicular fusion protein & 83 & P46460 & 1 \\
\hline PSD -95 & 80 & Q 62108 & 112 \\
\hline APPL adaptor protein & 80 & AF169797 & 8 \\
\hline Sarcolemmal associated protein-3 & 74 & Q 28623 & 3 \\
\hline GKAP/SAPAP & 74 & P97841 & 4 \\
\hline HSP70-like HS71 protein & 71 & U 73744 & 24 \\
\hline Kinesin light chain 2 & 67 & 088448 & 2 \\
\hline PP2A regulatory $\mathrm{R} 1 \alpha$ chain & 65 & P30153 & 1 \\
\hline CaM kinase II $\beta$ & 60 & P28652 & 1 \\
\hline PP2B (C alcineurin) $\alpha$ chain & 59 & P20652 & 3 \\
\hline$\alpha$-Internexin & 56 & P46660 & 2 \\
\hline CaM kinase $\| \alpha$ & 54 & P11798 & 4 \\
\hline RN A binding protein FUS/TLS & 53 & P35637 & 1 \\
\hline Tubulin $\alpha-4$ chain & 50 & P05215 & 3 \\
\hline Est736.26 & & A 1047568 & 2 \\
\hline Est700.75 & & A1428173 & 1 \\
\hline Est571.14 & & AA982950 & 1 \\
\hline Est762.20 & & AA 592427 & 1 \\
\hline
\end{tabular}

Visualized protein bands of 60-300 kD were prepared and analyzed as described (Methods and Fig. 2). Mr, relative molecular mass; number of peptides, total number of matching peptides sequenced by MS/MS for a specific protein. N ote that the number of peptides sequenced from each protein does not directly correlate to quantity of protein. Peptide lengths were 10-20 residues, and the peptides were unique and did not match any other sequences in the databases.

with NM DAR signaling and scaffolding (Table $\mathbf{1}$ ) and protein identification using mass spectrometry (Fig. 2 and Table 2). The protein bands indicated in Fig. 1g were analyzed by online LC-M S/MS (Fig. 2). It is important to note that thesestrategies do not show absolute levels and stoichiometry of subunits.

The N M DA and mGlu receptors have been implicated in the induction of synaptic plasticity through activation of second messenger pathways ${ }^{1,2}$. We therefore examined a wide range of different kinases and phosphatases in the NRC and found several specific serine-threonine kinase and phosphatase family members. We found protein kinase A (PKA) catalytic subunit and regulatory subunit $R 2 \beta$ ( $R 1, R 1 \alpha$ and $R 2 \alpha$ were not detected) and protein kinase C (PKC) isoforms $\beta, \gamma, \varepsilon$, but not $\alpha, \delta, \eta, \theta, \imath, \lambda$ isoforms. That a subset of a protein family associated with the $\mathrm{NRC}$, indicating specific association, was observed repeatedly for multiple classes of proteins in this study. The $\alpha$ and $\beta$ subunits of CaM KII were associated, as reported ${ }^{20}$, and also found to be in the phosphorylated active form. The presence of these kinases, which are involved in induction of synaptic plasticity, suggests that counterpart phosphatases may also be present in the NRC. We found that PP1 and PP2A were strongly associated and PP2B (calcineurin) and PP 5 were also associated. PKA and PP1 are linked to the NR1 subunit via an adapter protein (Yotiao) and can modulate NM DAR currents in vitro ${ }^{21}$. We found Yotiao and another PKA adaptor, AKAP150, suggesting that PKA may be linked to the NRC at multiple locations, perhaps in distinct pathways.

Because tyrosine phosphorylation is also involved with N M DAR ${ }^{22}$ and synaptic plasticity ${ }^{23}$, we examined cytoplasmic tyrosine kinases and found Src (but not Fyn) and the calciumactivated kinase Pyk2 (but not the Pyk2 homologue focal adhesion kinase, FAK) associated with the NRC. Although tyrosine phosphatase inhibitors influence N M DAR channels ${ }^{22}$, the identity of relevant tyrosine phosphatases has remained unclear. We 
detected PTP1D/SH P2, which associates with NR2B24, but neither the related PTP1B or PTP1C in the NRC, implicating this tyrosine phosphatase in $N R C$ regulation.

How N M DAR-mediated kinase and phosphatase signal integration occurs is unclear, but the involvement of Ras, which integrates downstream signals in other cellular contexts, is suggested by the altered plasticity and learning in mice with mutations in modulators of Ras (Ras-GRF ${ }^{25}$ or NF1; ref. 26). We found both $\mathrm{H}$-Ras and Rap2 small G proteins and their GTPase activating proteins NF1 and SynGAP (which binds PSD-95), but not p120GAP in the NRC. Rap1, which may couple PKA to c-Raf1, was not detectable in the NRC. Ras mediates its effects by differential activation of several downstream effector pathways ${ }^{27}$, including the c-Raf1-M EK-ERK/M APK pathway, PI 3 kinase and RalA. The different MAPK pathways are organized into modules composed of the key enzymes producing that cascade, tethered within a cell to produce signal specificity ${ }^{28}$. This was al so the case for the NRC, as ERK 1 and ERK 2 and their upstream activating kinases MEK 1, MEK2 and C-Raf1 were found, but not JNKK 1/M KK 4 and ERK 3. Moreover, the ERK phosphatase (MKP2), which inactivates ERK, was also identified. In addition to ERK/M APK pathway, mass spectrometry identified APPL ${ }^{29}$, an adaptor protein linking the $\mathrm{p} 110$ subunit of PI3 kinase to AKT/PKB kinase. These observations that ERK/M APK and the other Ras effectors, PI 3 kinase and Ral were detected along with adaptors and regulators indicates that glutamate receptors drive Ras, which is coupled to distinct downstream pathways organized as modules within the NRC.

The role of ERK/M APK pathways in synaptic plasticity and learning has received considerable attention because phosphorylation of ERK accompanies these processes, which are also disrupted by inhibitors of $\mathrm{MEK}^{30}$. Phosphorylation of ERK s has mainly been implicated in regulating transcription, through phosphorylation of Rsk-2, which translocates to the nucleus to phosphorylate transcription factors CREB and CREM . Interestingly, phospho-ERK was not present in the NRC, although it was readily detected in the extract, consistent with its ability to translocate from the NRC on phosphorylation. M oreover, Rsk-2 was found within the NRC, although the transcription factors CREB and CREM were not detectable. We noticed that several of the NRC proteins (Homer, NR1, NR2B, PKC $\gamma$, ERK2, C-Raf1, HSP 70) are encoded by activity-dependent genes, and we therefore tested Arg3.1/Arc 31,32 , a postsynaptic protein of unknown function that is also rapidly regulated by LTP. Arg3.1 was readily detected in the NRC, suggesting it may participate in signaling and dynamic organization of the NRC. Thus the NRC contains signaling mechanisms that could contribute to transcriptional activation following synaptic activation, and the N RC could then itself undergo structural changes secondary to altered gene expression. Another potential ERK effector, CPLA ${ }^{33}$, is also regulated by $\mathrm{Cit}$ ron, which binds PSD -95. M ice lacking CPLA2 show resistance to ischemic neuronal damage ${ }^{34}$, and CPLA2 generates arachidonic acid, a candidate transynaptic retrograde signaling molecule.

Changes in synapse structure may be fundamental to the storage of long-term memory, and roles for cell-adhesion molecules and cytoskeletal structural proteins in this process are suggest$\mathrm{ed}^{35}$. M any of the signaling proteins described above are known regulators of cytoskeleton and cell adhesion. Actin cytoskeleton is involved in NMDAR channel properties ${ }^{36}$, NMDAR-mediated LTP $^{37}$ and NM DAR localization ${ }^{38}$, and is dynamically regulated in spines by neural activity ${ }^{39}$. We found $\alpha$-actinin 2 and spectrin (Fodrin), which bind NMDAR subunits ${ }^{40}$, and actin-binding proteins, including cortactin, cortactin-binding proteins
(CortBP1 and Shank) and M AP2, but not Ezrin, Tensin or Vinculin, in the NRC. Surprisingly, the cell-adhesion proteins NCadherin and Desmoglein, two cadherin family members and their cytoplasmic interacting proteins $\beta$-caten in, ZO- 1 and p120cas were detected, although E-cadherin, P-cadherin, cadherin-5, $\alpha$-catenin and $\gamma$-catenin were negative. L1 adhesion protein, which is required for learning and synaptic plasticity ${ }^{41}$, was also detected in the NRC. These adhesion proteins may be involved in structural organization of the NRC at the synapse; however, the involvement of cadherins ${ }^{42}$ and $L 1$ in synaptic plasticity ${ }^{41}$ make it tempting to speculate that this glutamate receptor-cell-adhesion protein complex could provide multiple trans-synaptic signaling pathways, whereby adhesion-mediated signaling is coupled to transmitter signaling mechanisms.

M ass spectrometry of large-scale isolated N M DAR complexes has confirmed and extended the identification of NRC components beyond yeast 2-hybrid assays and immunoblotting (Tables 1 and 2). Some N RC components were not detected by MS, which may be due to the low levels of protein in the selected gel fragments. In addition to identification of previously unsuspected known proteins, the M S approach also identified matching peptides that are represented in the dbEST database, indicating novel proteins in the NRC. During the course of this work, as the databases expanded, several EST matches were subsequently found to match known proteins. For example, the peptide sequence DLKEI LTLK (Fig. 2d) was originally identified as mouse hippocampus EST (AV153731), and later a report of the full-length CDNA sequence allowed us to match with seven other peptides, thus identifying this NRC protein as APPL, a novel adaptor for $\mathrm{PI} 3$ kinase and $\mathrm{AKT} / \mathrm{PKB}^{29}$. Although it is clear that some protein complexes contain dozens of proteins ${ }^{11,12}$, the large number of NRC proteins identified in this study does not imply that all proteins exist in a single homogeneous complex. Heterogeneity could result from different complexes in the starting material, as may be found at different synapses. M oreover, the large size of the complex may reflect dynamic assembly, perhaps involving activity-dependent genes, or transient protein-protein interactions of signaling complexes, which is a feature of many signaling pathways involving the proteins in the $\mathrm{NRC}^{6}$.

\section{Discussion}

The proteomic approach complements and extends studies using yeast two-hybrid screens for the identification of proteins in neurotransmitter receptor complexes. Fourteen proteins that interact with NR1 and NR2, as determined by two-hybrid screens, also coimmunoprecipitate with NM DAR from brain (PSD-95, Chapsyn110/PSD-93, SAP102, GKAP/SAPAP, Yotiao, SynGAP, Calmodulin, nNOS, PI3-kinase, Citron, MAP2B, actin, $\alpha$-actinin2, spectrin). Twelve proteins interact with these 14 proteins, but were not formally shown to exist in NRCs (mGluR, GluR6/7, Homer/Vesl1, Shank, PKA-R2 $\beta$, PP1, PKA catalytic subunit, PLC $\gamma$, Cortactin, CortBP-1, phosphofructokinase, APPL). Four proteins coimmunoprecipitate with NM DARs without defined interaction domains (CaM KII, Src, PTP1D/SH P2, tubulin). All 30 of these proteins were detected in our analysis, including PI 3 kinase, which interacts via SH 2 phosphorylation with NR2 and therefore escapes detection in two-hybrid screens. We identified a third group of 19 proteins, not previously known to be structurally linked to NRCs, but functionally implicated in NM DAR-dependent synaptic plasticity, including PKC $(\beta, \gamma, \varepsilon)$, PP2A, PP2AR1 $\alpha$, PP2B, NF1, Ras, Rap2, C-Raf1, M EK 1/2, ERK1/2, M KP2, Rsk-2, CPLA2, N-cadherin, L1, Arg3.1/Arc and HSP70. A fourth set of six proteins that were not structurally or 


\section{articles}

functionally linked to NMDAR but are known to interact with the above proteins include $\beta$-Catenin, Z0-1, pp120CAS, myosin, Clathrin and Dynamin. There remains a set of 19 proteins, including 5 ESTs, Pyk2, AKAP150, PP5, Rac1, RalA, NSF, SAP97, Desmoglein, Bassoon, P53 binding protein, Sarcolemmal associated protein-3, Kinesin light chain, RNA binding protein FUS/TLS and $\alpha$-internexin. Interestingly, this latter set includes proteins that might be involved in postsynaptic receptor trafficking and spine translational regulation.

The structure of the NRC isolated from the mouse brain indicates that subsets of neurotransmitter receptors, cell-adhesion proteins, adapters, second messengers and cytoskeletal proteins are organized together into a physical unit comprising signaling pathways. Several features of the NRC provide insights into the specific functions of this complex, particularly in the physiological context of NM DAR-dependent synaptic plasticity. The simplest general function for this NRC may bein the 'induction phase' of synaptic plasticity in contrast to the 'expression phase' as described in electrophysiological experiments. Induction involves activation of the NM DAR and mGluR, $\mathrm{Ca}^{2+}$ influx and second messenger signaling and occurs within the first hour after the train of stimuli. Expression or maintenance of synaptic plasticity after one hour is resistant to inhibition of NM DAR or second messengers and is mediated by AM PA receptors. Thus the NRC contains the molecular machinery for the induction of plasticity, and AM PA receptor complexes are separate entities mediating expression. The assembly of specific signaling modules composed of effector pathways (for example, Ras-ERK/M AP or Ras-PI3 kinase) might regulate specific components of LTP or LTD. In addition to trafficking and phosphorylation of AM PA receptors, the expression of plasticity is reported to involve multiple cellular mechanisms, including cytoskeletal changes in spine structure, local translation regulation and gene transcription. These functions could be linked to the NM DA receptor via the NRC proteins.

This model of the NRC as an induction device is supported by targeted mouse mutations, transgenic expression and pharmacological inhibition of NRC proteins that alter the induction of synaptic plasticity. Induction of synaptic plasticity is altered by targeted mouse mutations in NR1, NR2A, mGluR1, PSD-95, PKA catalytic and regulatory subunits, PKC $\gamma, \mathrm{CaMKII}, \mathrm{nN} O \mathrm{~S}$, NF1 or H-Ras, by transgenic expression of NR2B, CaM KII, PP2B, PKA inhibitors or $L 1$, and by pharmacological inhibition of NM DAR, mGluR, PKA, PKC, CaM KII, MEK, tyrosine kinases, PP1, PP2A, PP2B, Cadherins, L1, actin polymerization, Calmodulin, nN OS, PI3 kinase or CPLA2. A physiological parallel with these disruptions in NRC proteins is observed during postnatal development of the hippocampus and cortex. The threshold for N M DAR-mediated LTP and LTD is age dependent ${ }^{2}$, and during this time the levels of expression of many NRC proteins and their association into N M DAR complexes changes ${ }^{43}$. The NRC is also well suited to induce bidirectional synaptic plasticity (LTP and LTD) because many calcium-sensitive proteins with kinases and phosphatases are beneath the NM DAR calcium pore in a microdomain. The composition of this complex goes some way to addressing questions raised 5 regarding the apparent diversity of molecules involved with synaptic plasticity, as a substantial subset of these molecules are components of the NRC and map onto the function of induction.

In addition to a rolein synaptic plasticity, the NRC described here is likely to be important for cognitive function and in particular learning and memory. Learning impairments in rodents are associated with genetic and pharmacological disruption of multiple components of the NRC (NR1, NR2A, NR2B, PSD-95,
PKA subunits, PKC isoforms, CaM KII, PP1, PP2A, PP2B, MEK, NF1, nNOS, CPLA2 and L1). Although these data support a role for the NRC in both learning and synaptic plasticity, it may be premature to conclude that synaptic plasticity is itself required for learning, as the NRC seems to comprise multiple effector pathways, which may be differentially required for learning or plasticity or other cellular processes ${ }^{44}$. Because the term NRC was used to describe the channel formed by N M DAR subunits, 'Hebbiasome' or 'potentiosome' are alternative terms describing the function of the described complexes in controlling synaptic potential and $\mathrm{Hebbian}$ synaptic properties.

We were surprised to find that three of the proteins (NF1, ref. 45; L1, ref. 46; Rsk-2, ref. 47) detected in this proteomic study wereencoded by genes that underlie human mental retardation and learning impairment. This implies that the defect in these patients is in the function of the NRC. Moreover, these findings suggest that pharmacological manipulation of the NRC, as distinct to NMDARs alone, may provide new therapeutic avenues for these patients. Proteomic tools combined with genetic dissection of the NRC signaling pathways in mutant mice should lead to an understanding of the specific contributions of each pathway to the cellular mechanisms of synaptic plasticity and learning, as well as provide a new route toward understanding human neuropsychiatric conditions.

\section{Methods}

Antibodies. Affinity-purified NR1-specific sheep polyclonal antibodies (MAP-NR1) were generated using a multiple-antigen peptide (MAP) of the last 20 amino acids of NR1 ((H-RRAIEREEGQ LQLCSRH RES) 8M AP; Diagnostics Scotland, Carluke, UK). Antibody sources (column $A b$ in Table 1): $a$, J.H. Morrison; b, Upstate Biotech, Waltham, Massachusetts; c, Chemicon, Wealdstone, UK; d, Pharmingen, San Diego, California; e, R. Huganir; f, Transduction Laboratories, Lexington, Kentucky; g, M. Watanabe; h, Alomone Labs, Jerusalem, Israel; i, H. Kreienkamp; j, K. Inokuchi; k, E. Ziff; I, J. Scott; m, J. Henley; $n$, Promega, Madison, Wisconsin; o, P. Cohen; $p$, Oncogene, Nottingham, UK; q, N ew England Biolabs, Hitchin, UK; r, Santa Cruz Biotechnology, Santa Cruz, California; s, Calbiochem, Nottingham, UK; t, D. Kuhl; u, D. Coleman; v, Sigma, Poole, UK; w, RocheM olecular Biochemicals, Lewes, UK; $x$, J. Parsons.

Purification of the NMDAR complex. The receptor complex was isolated from mouse forebrain extracts using covalently coupled M AP-NR1 immunoaffinity resins, immunoprecipitation with the same antibody, or peptide affinity chromatography with a hexapeptide of the NM DA-R2B C-terminus (SIESDV; H.H . and S.G.N.G., unpublished data). In brief, samples were homogenized in $1 \%(\mathrm{w} / \mathrm{v})$ deoxycholate-containing buffer at pH 9.0, spun for 30 minutes at $50.000 \times g$ at $4{ }^{\circ} \mathrm{C}$, followed either by incubation with M AP-NR1 antibody and subsequent protein G-sepharose precipitation, or by immunoaffinity chromatography using M AP-NR1 antibody-substituted affigel-10 (BioRad, Hemel Hempstead, UK; $5 \mathrm{mg}$ antibody per $\mathrm{ml}$ resin), or the NR2B peptide resin ( $5 \mathrm{mg}$ peptide per $\mathrm{ml}$ affigel-10 resin). The resins were washed after an overnight incubation at $4^{\circ} \mathrm{C}$ with 100 to 1000 column volumes of extraction buffer at 4으, and proteins were separated from the resin by boiling in 4\% SDS for 30 minutes.

Western blotting. Samples were separated by SDS-PAGE and transferred to PVDF membrane at 4을 $\mathrm{C}$ for 90 minutes at $75 \mathrm{~V}$ in $10 \%(\mathrm{v} / \mathrm{v})$ methanol and $10 \mathrm{mM}$ CAPS, pH 11.0. Dilution of primary antibodies was between $1: 100$ and $1: 1000$ depending on the quality of the IgG. Signals were detected using peroxidase-linked secondary IgGs and enhanced chemiluminescence.

Mass spectrometry sample preparation. N M DAR complex samples were separated by SDS-PAGE and stained by Coomassie blue or silver staining $^{48}$. Individual protein bands of 60-300 kD were excised, reduced, alkylated and digested with trypsin ${ }^{49}$. The resultant peptide mixtures were 
analyzed by on-line liquid chromatography tandem mass spectrometry (LC-MS/MS) to generate peptide sequence information ${ }^{50}$.

Online LC-MS/MS analysis. Chromatographic separations of the peptide mixture were done on a $180 \mu \mathrm{m}$ PepM ap column using an Ultimate LC system (LC Packings, Amsterdam, Netherlands) delivering a gradient to formic acid $(0.05 \%)$ and acetonitrile. The eluting peptides were ionized by electrospray ionization on a Q-TOF hybrid mass spectrometer (Micromass, Wythenshawe, UK) fitted with a Z-spray source. The instrument, in automated switching mode, selects precursor ions based on intensity for peptide sequencing by collision-induced fragmentation tandem MS. The M S/M S analyses were conducted using collision energy profiles that were chosen based on the $\mathrm{m} / \mathrm{z}$ of the precursor; a total of nine M S/M S scans were acquired per precursor. Several hundred M S/M S spectra were generated per run, allowing the analysis of complex mixtures without any prior interpretation. The mass spectral data was processed into peak lists containing $\mathrm{m} / \mathrm{z}$ value, charge state of the parent ion, fragment ion masses and intensities, and correlated with proteins and nucleic acid sequence databases using $M$ ascot software ( $M$ atrix Science, London, UK). Proteins were identified based on matching the $\mathrm{MS} / \mathrm{MS}$ data with mass values calculated for selected ion series of a peptide. A non-redundant protein database and a nucleotide database (dbEST) were searched without applying any constraints on molecular weight or species. M ost proteins were identified with several peptide matches, although a few were assigned on the basis of a single peptide provided near-complete peptide sequence had been obtained.

Note: A completelist of references for all protein interactionscan befound on the Nature Neuroscienceweb site (http://www.nature.com/neuro/web_specials/).

\section{ACKNOWLEDGEMENTS}

Antibodies were provided by those listed in M ethods. Wethank T.J. O'D ell and P. Brophy for comments. H.H. and S.G. were supported by the Wellcome Trust.

\section{Received 14 April; Accepted 23 May 2000}

1. Bliss, T. V. \& Collingridge, G. L. A synaptic model of memory: long-term potentiation in the hippocampus. Nature 361, 31-39 (1993).

2. Bear, M. F. \& Malenka, R. C. Synaptic plasticity: LTP and LTD. Curr. Opin. Neurobiol. 4, 389-399 (1994).

3. Heresco-Levy, U. \& Javitt, D. C. The role of N-methyl-D-aspartate (NM DA) receptor-mediated neurotransmission in the pathophysiology and therapeutics of psychiatric syndromes. Eur. Neuropsychopharmacol. 8, 141-152 (1998).

4. Hollmann, M. \& Heinemann, S. Cloned glutamate receptors. Annu. Rev. Neurosci. 17, 31-108 (1994).

5. Sanes, J. R. \& Lichtman, J. W. Can molecules explain long-term potentiation? Nat. Neurosci. 2, 597-604 (1999).

6. Pawson, T. \& Scott, J. D. Signaling through scaffold, anchoring, and adaptor proteins. Science 278, 2075-2080 (1997).

7. Kim, J. H. \& Huganir, R. L. Organization and regulation of proteins at synapses. Curr. Opin. Cell Biol. 11, 248-254 (1999).

8. H sueh, Y. P. \& Sheng, M. Anchoring of glutamate receptors at the synapse. Prog. Brain Res. 116, 123-131 (1998).

9. Kornau, H. C., Seeburg, P. H. \& Kennedy, M. B. Interaction of ion channels and receptors with PDZ domain proteins. Curr. Opin. Neurobiol. 3, 368-373 (1997).

10. M igaud, $M$. et al. Enhanced long-term potentiation and impaired learning in mice with mutant postsynaptic density- 95 protein. Nature 396, 433-439 (1998).

11. Mendelsohn, A. R. \& Brent, R. Protein interaction methods-toward an endgame. Science 284, 1948-1950 (1999).

12. Neubauer, G. et al. M ass spectrometry and EST-database searching allows characterization of the multi-protein spliceosome complex. Nat. Genet. 20, 46-50 (1998).

13. Hisatsune, C., Umemori, H., M ishina, M . \& Yamamoto, T. Phosphorylationdependent interaction of the N-methyl-D-aspartate receptor epsilon 2 subunit with phosphatidylinositol 3-kinase. Genes Cells 4, 657-666 (1999).

14. Craven, S. E., El-Husseini, A. E. \& Bredt, D. S. Synaptic targeting of the postsynaptic density protein PSD-95 mediated by lipid and protein motifs. Neuron 22, 497-509 (1999).

15. Blackstock, W. P. \& Weir, M. P. Proteomics: quantitative and physical mapping of cellular proteins. Trends Biotechnol. 17, 121-127 (1999).

16. O'Brien, R. J., Lau, L. F. \& Huganir, R. L. M olecular mechanisms of glutamate receptor clustering at excitatory synapses. Curr. O pin. Neurobiol. 8, 364- 369 (1998).
17. Garcia, E. P. et al. SAP90 binds and clusters kainate receptors causing incomplete desensitization. Neuron 21, 727-739 (1998).

18. Naisbitt, S. et al. Shank, a novel family of postsynaptic density proteins that binds to the N M DA receptor/PSD-95/GKAP complex and cortactin. Neuron 23, 569-582 (1999).

19. Tu, J. C. et al. Coupling of mGluR/Homer and PSD-95 complexes by the Shank family of postsynaptic density proteins. Neuron 23, 583-592 (1999).

20. Leonard, A. S., Lim, I. A., Hemsworth, D. E., Horne, M. C. \& Hell, J. W. Calcium/calmodulin-dependent protein kinase II is associated with the $\mathrm{N}$-methyl-D-aspartate receptor. Proc. Natl. Acad. Sci. USA 96, 3239-3244 (1999).

21. Westphal, R. S. et al. Regulation of NMDA receptors by an associated phosphatase-kinase signaling complex. Science 285, 93- 96 (1999).

22. Wang, Y. T. \& Salter, M. W. Regulation of NMDA receptors by tyrosine kinases and phosphatases. Nature 369, 233-235 (1994).

23. O'D ell, T. J., Kandel, E. R. \& Grant, S. G. Long-term potentiation in the hippocampus is blocked by tyrosine kinase inhibitors. Nature 353, 558-560 (1991).

24. Lin, S. Y. et al. Brain-derived neurotrophic factor enhances association of protein tyrosine phosphatase PTPID with the NMDA receptor subunit NR2B in the cortical postsynaptic density. M ol. Brain Res. 70, 18-25 (1999).

25. Brambilla, R. et al. A role for the Ras signalling pathway in synaptic transmission and long- term memory. Nature 390, 281-286 (1997).

26. Silva, A. J. et al. A mouse model for the learning and memory deficits associated with neurofibromatosis type I. Nat. Genet. 15, 281-284 (1997).

27. Gille, H.\& Downward, J. Multiple ras effector pathways contribute to $G(1)$ cell cycle progression. J. Biol. Chem. 274, 22033-22040 (1999).

28. Schaeffer, H. J. \& Weber, M. J. M itogen-activated protein kinases: specific messages from ubiquitous messengers. M ol. Cell. Biol. 19, 2435-2444 (1999).

29. M itsuuchi, Y. et al. Identification of a chromosome 3p14.3-21.1 gene, APPL, encoding an adaptor molecule that interacts with the oncoproteinserine/threonine kinase AKT2. Oncogene 18, 4891-4898 (1999).

30. Impey, S., Obrietan, K. \& Storm, D. R. Making new connections: role of ERK/M AP kinase signaling in neuronal plasticity. Neuron 23, 11-14 (1999).

31. Link, W. et al. Somatodendritic expression of an immediate early gene is regulated by synaptic activity. Proc. Natl. Acad. Sci. USA 92, 5734-5738 (1995).

32. Lyford, G. L. et al. Arc, a growth factor and activity-regulated gene, encodes a novel cytoskeleton-associated protein that is enriched in neuronal dendrites. Neuron 14, 433-445 (1995)

33. Lin, L. L. et al. CPLA2 is phosphorylated and activated by MAP kinase. Cell 72, 269-278 (1993).

34. Bonventre, J. V. et al. Reduced fertility and postischaemic brain injury in mice deficient in cytosolic phospholipase A2. N ature 390, 622-625 (1997).

35. Murase, S. \& Schuman, E. M. The role of cell adhesion molecules in synaptic plasticity and memory. Curr. O pin. Cell Biol. 11, 549-553 (1999).

36. Rosenmund, C.\& Westbrook, G. L. Calcium-induced actin depolymerization reduces N M DA channel activity. Neuron 10, 805-814 (1993).

37. Kim, C. H. \& Lisman, J. E. A role of actin filament in synaptic transmission and long-term potentiation. J. Neurosci. 19, 4314-4324 (1999).

38. Allison, D. W., Gelfand, V. I., Spector, I. \& Craig, A. M. Role of actin in anchoring postsynaptic receptors in cultured hippocampal neurons: differential attachment of NMDA versus AM PA receptors. J. Neurosci. 18, 2423-2436 (1998)

39. Fischer, M., Kaech, S., Knutti, D. \& M atus, A. Rapid actin-based plasticity in dendritic spines. Neuron 20, 847-854 (1998).

40. Wechsler, A. \& Teichberg, V. I. Brain spectrin binding to the N M DA receptor is regulated by phosphorylation, calcium and calmodulin. EM BO J. 17, 3931-3939 (1998).

41. Luthl, A., Laurent, J. P., Figurov, A., M uller, D. \& Schachner, M . H ippocampal long-term potentiation and neural cell adhesion molecules L1 and NCAM . Nature 372, 777-779 (1994).

42. Tang, L., Hung, C. P. \& Schuman, E. M. A role for the cadherin family of cell adhesion molecules in hippocampal long-term potentiation. Neuron 20, 1165-1175 (1998)

43. Sans, N., Petralia, R. S., Wang, Y. X., Blahos, J., Hell, J. W. \& Wenthold, R. J. A developmental change in NMDA receptor-associated proteins at hippocampal synapses. J. Neurosci. 20, 1260-1271 (2000).

44. Grant, S. G. N. in Handbook of M olecular-Genetic Techniques for Brain and Behavior Research (eds. Crusio, W. E. \& Gerlai, R. T.) 315-328 (Elsevier, Amsterdam, 1999).

45. Ozonoff, S. Cognitive impairment in neurofibromatosis type 1. Am. J. M ed. Genet. 89, 45-52 (1999).

46. Fransen, E., Van Camp, G., D'Hooge, R., Vits, L. \& Willems, P. J. Genotypephenotype correlation in L1 associated diseases. J. M ed. Genet. 35, 399-404 (1998).

47. Merienne, K. et al. A missense mutation in RPS6KA3 (RSK2) responsible for non-specific mental retardation. Nat. Genet. 22, 13-14 (1999).

48. Shevchenko, A., Wilm, M., Vorm, O. \& Mann, M. Mass spectrometric sequencing of proteins silver-stained polyacrylamide gels. Anal. Chem. 68, 850-858 (1996)

49. Wilm, M. et al. Femtomole sequencing of proteins from polyacrylamide gels by nano-electrospray mass spectrometry. Nature 379, 466-469 (1996).

50. Link, A. J. et al. Direct analysis of protein complexes using mass spectrometry. Nat. Biotechnol. 17, 676-682 (1999). 